\title{
Ground-based direct detection of close-in extra-solar planets with nulling and high order adaptive optics
}

\author{
M. Langlois ${ }^{1}$, A. Burrows ${ }^{2}$, and P. Hinz ${ }^{2}$ \\ 1 Laboratoire d'Astophysique de Marseille, 2 place Le Verrier, 13248 Marseille, France \\ e-mail: maud. langlois@oamp.fr \\ 2 Steward Observatory, Tucson, AZ 85719, USA
}

Received 17 November 2004 / Accepted 11 April 2005

\begin{abstract}
Ground-based direct detection of extra-solar planets is very challenging due to high planet to star brightness contrasts. For giant close-in planets, such as have been discovered by the radial velocity method, closer than $0.1 \mathrm{AU}$, the reflected light is predicted to be fairly high yielding a contrast ratio ranging from $10^{-4}$ to $10^{-5}$ at near infra-red wavelengths. In this paper, we investigate direct detection of reflected light from such planets using nulling interferometry, and high-order adaptive optics in conjunction with large double aperture ground-based telescopes. In this configuration, at least $10^{-3}$ suppression of the entire stellar Airy pattern with small loss of planet flux as close as 0.03 arcsec is achievable. Distinguishing residual starlight from the planet signal is achieved by using the center of gravity shift method or multicolor differential imaging. Using these assumptions, we derive exposure times from a few minutes to several hours for direct detection of many of the known extra-solar planets with several short-baseline double aperture telescopes such as the Large Binocular Telescope (LBT), the Very Large Telescope (VLT) and the Keck Telescope.
\end{abstract}

Key words. stars: planetary systems - instrumentation: interferometers - instrumentation: high angular resolution instrumentation: adaptive optics

\section{Introduction}

Extra-solar planets have been hunted for many years by several groups mainly by the radial velocity technique to detect the motion of the parent star using very sensitive spectroscopic observations of the Doppler shift. It is only since the first extra-solar planet discovery around 51 Pegasi (Mayor et al. 1995) that confirmed detections of more than 100 planetary candidates have been obtained (Marcy \& Butler 1997; Mayor \& Queloz 1995). The detected planetary companions are quite massive $0.16 M_{\mathrm{J}}<M \sin (i)<13 M_{\mathrm{J}}$ and very close to their primary stars (0.03-4 AU). Recently, for the first time an extra-solar planet has been observed transiting its star, HD 209458 (Henry et al. 2000). This discovery was confirmed by the detection by the radial velocity's method and allowed determination of both radius and mass $\approx\left(1.42 R_{\mathrm{J}}, 0.69 M_{\mathrm{J}}\right)$, both complying gas giant planets. Also the first attempt at direct detection of the Dopplershifted starlight reflected from the planet orbiting $\tau$ Boötis by Cameron et al. (1999) lead to an upper limit to the albedo and radius. Direct detection of an extra-solar planet's emitted or reflected light, coupled with broadband spectroscopy, would provide complementary information on the planet's characteristics such as its temperature, chemical composition, and the presence of chemical tracers associated with life. However, due to planet's faintness and its location very close to the parent star, the technical requirements are at the theoretical limits of performance and, hence, very challenging. Ground-based imaging or spectroscopy requires very high contrast, achievable only by using high-order adaptive optics (AO) to reduce the light scattered by atmospheric turbulence, such as described in Angel (1994); Stahl \& Sandler (1995); Langlois et al. (2003) and by using in addition a coronograph (Malbet 1996; Beuzit et al. 1997; Roddier \& Roddier 1997, Baudoz et al. 2000; Rouan et al. 2000) or a nulling instrument (Bracewell 1978; Woolf \& Angel 1997; Hinz et al. 1998, 1999) to reduce the diffracted stellar light.

In this paper, we consider the case of double AO corrected aperture telescopes using the Bracewell nulling technique. We estimate the efficiency of this technique, the quality of the adaptive optics correction, the planet to star brightness contrast, and we derive detection limits for known close-in planets assuming either detection of the center of gravity shift or the use of the spectral difference imaging method to distinguish the planet from its hosting star.

\section{Planet reflected starlight flux}

The planets detectability is driven by the amount of light they emit or reflect. For very close planets, visible and near-infrared fluxes are dominated by reflected starlight as discussed in 
Seager et al. (2000) and Sudarsky et al. (2003). For a given age and mass, beyond a certain separation greater than considered here $(S \geq 0.3 \mathrm{AU})$ and depending on the wavelength and the star spectral type, the intrinsic luminosity of the planet takes over. The ratio, $\epsilon$, of the flux reflected by the planet is given by Brown \& Burrows (1990): $\epsilon=\phi(\alpha)\left(\frac{R_{\mathrm{p}}}{S}\right)^{2} p$, where $R_{\mathrm{p}}$ is the planet radius, $p$ is the geometric albedo defined as the planet flux divided by the reflected flux from a perfectly diffusing disk of the same radius, and $\phi(\alpha)$ is the phase function. The phase function varies from 1.0 to 0.0 for $\alpha$ from $0^{\circ}$ to $180^{\circ}$. When $\alpha=90^{\circ}$, maximum angular separation, typical phase functions are 0.25 for a cloudy planet and 0.1 for a rocky planet (Irvine 1968; Lane \& Irvine 1973). For simplicity, we use a phase value equal to 0.3 in the following calculations. The geometric albedo, $p$, of a perfectly conservative Lambert sphere is $2 / 3$. For a real planet, it can be higher due to backscattering or lower due to surface absorption. The albedo has been estimated by Seager et al. (2000) for close-in giant planets and range from 0.0013 to 0.44 for $10 \mu \mathrm{m}$ particles with a strong backscattering peak. In comparison, higher albedo values ranging from 0.02 to 0.8 have been found by Sudarsky et al. (2000). In our solar system typical values range from 0.4 to 0.2 for cloudy or rocky planets. In the following, to represent average conditions, we use an albedo of 0.5 .

The reflected flux also depends on the planets radii, a parameter that depends on age, composition and mass. Based on Hubbard (1984) the maximum radius of a planet, in fact of any object less massive than one tenth of a solar mass, is $1.13 R_{\mathrm{J}}$. More recently it has been shown by Burrows et al. (2000) that close-in giant planets can be $20 \%-80 \%$ larger Jupiter due to stellar insulation. For example, $\tau$ Boo b's radius has been modeled to be between 1.6-1.8 $R_{\mathrm{J}}$. These theoretical predictions have been verified by the observation of the transit by HD 209458b of its primary where the radius was found by Mazeh et al. (2000) to be around $1.4 R_{\mathrm{J}}$. In this paper, we assume that planet radii range from 1 to $1.7 R_{\mathrm{J}}$ as given in Table 2 for several known planets. The planet-star contrast ratio estimated by Sudarsky et al. (2003) ranges from $10^{-7}$ to $10^{-4}$ for six known close-in planets except GJ876c as shown in Fig. 1. In all cases, the most favorable wavelength for detecting these planets appears to be between 1 and $1.5 \mu \mathrm{m}$ where both the flux and atmospheric transmission are high, the diffraction allows high resolution and strong absorption bands are present which could be used to discriminate the planet from the star. In the following we concentrate on detection at $1.3 \mu \mathrm{m}$ using partial Bracewell Nulling.

\section{Bracewell nulling efficiency at $1.3 \mu \mathrm{m}$}

The nulling technique used to remove star light is based on Bracewell (1978) interferometric methods where two apertures provide two stellar wavefronts exactly out of phase with no relative tilt, so there is perfect cancellation of the stellar light across the whole pupil while the companion signal remains unaffected. The transmission, $T$, for monochromatic light, is one of finely spaced fringes given by $T(\theta)=\sin ^{2} \frac{\pi \theta B}{\lambda}$, where $B$ is the interferometer baseline and $\theta$ is the angular distance from a line on the sky through the star perpendicular to the

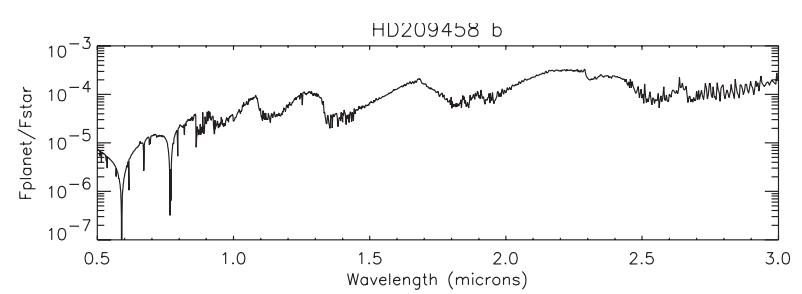

HD 83443 b
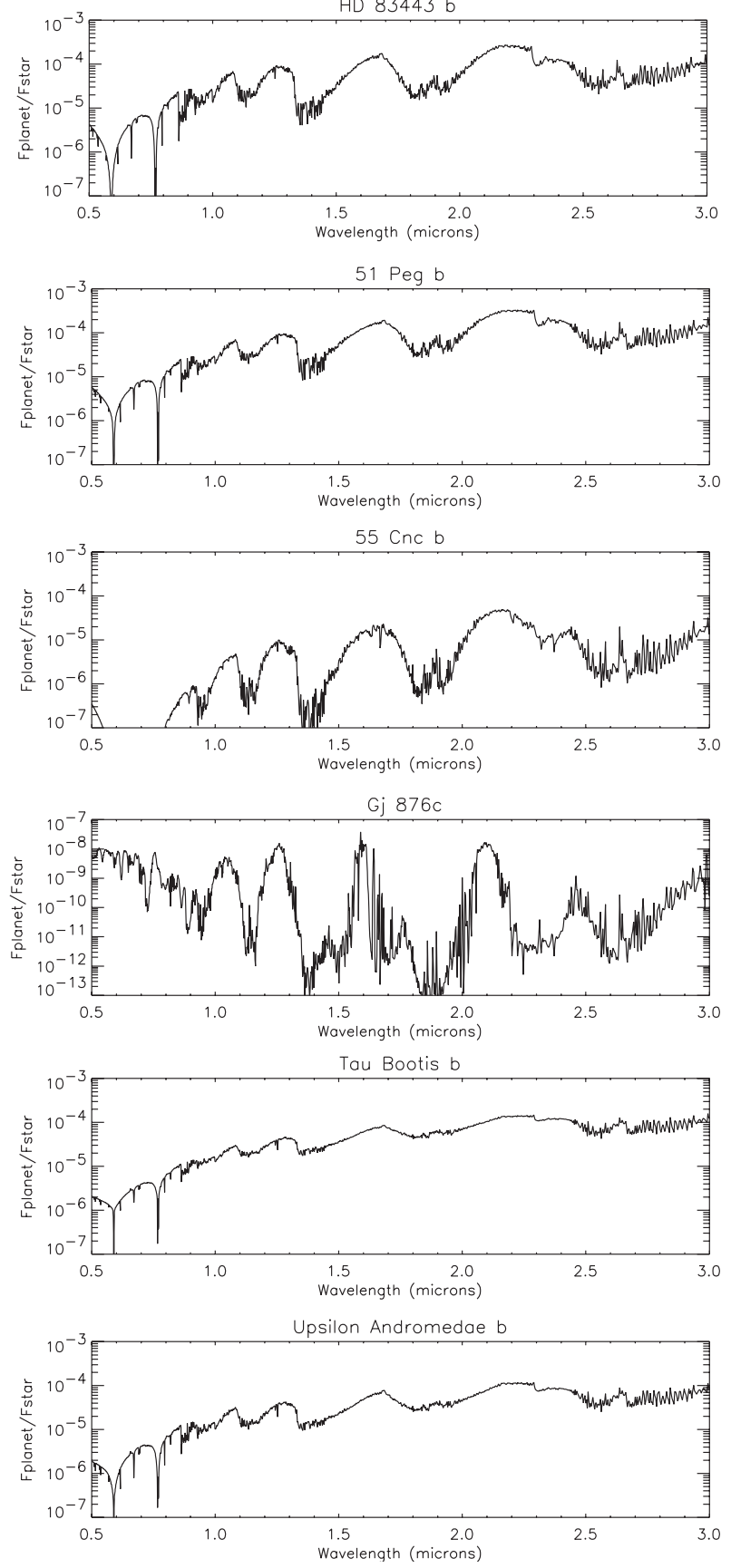

Fig. 1. The phase averaged planet-to-star flux contrast as a function of wavelength for seven known extrasolar planets from Sudarsky et al. (2003).

interferometer baseline. The extended parent star angular diameter results in imperfect starlight cancellation requiring the use of either differential wavelength imaging or the astrometric center of gravity method in order to distinguish the planet from 
Table 1. The minimum planet-star separation $\left(S_{\min }\right)$ versus stellar type and radius for leakage ratios smaller than $5 \times 10^{-4}$ in both astronomical units and milli-arcseconds, assuming a distance of 10 parsecs for the system.

\begin{tabular}{ccccc}
\hline \hline Type & $R_{\mathrm{s}} / R_{\odot}$ & $R_{\mathrm{s}}(\mathrm{AU})$ & $S_{\min }(\mathrm{AU})$ & $S_{\min }^{10 \mathrm{pc}}(\mathrm{mas})$ \\
\hline M5 & 0.27 & 0.0012 & 0.044 & 4.4 \\
M0 & 0.60 & 0.0028 & 0.098 & 9.8 \\
G5 & 0.95 & 0.0044 & 0.16 & 15.5 \\
G0 & 1.1 & 0.0051 & 0.18 & 17.8 \\
F5 & 1.3 & 0.0060 & 0.21 & 21.2 \\
F0 & 1.4 & 0.0065 & 0.23 & 22.8 \\
\hline
\end{tabular}

the stellar leak. The ratio of stellar flux leaking through the null, the rejection factor $R\left(\theta_{\mathrm{s}}\right)$, ultimately depends on the ratio of stellar angular diameter to the destructive fringes width $\left(\frac{\lambda}{B}\right)$. This factor, directly estimated by integrating the transmission over the stellar disk angular size, is given by

$R\left(\theta_{\mathrm{s}}\right)=\frac{4}{\pi \theta_{\mathrm{s}}} \int_{0}^{\theta_{\mathrm{s}}} \sin ^{2} \frac{\pi \theta B}{\lambda} \sqrt{1-\left(\frac{\theta}{\theta_{\mathrm{s}}}\right)^{2}} \mathrm{~d} \theta$,

where $\theta_{\mathrm{s}}$ is the star disk radius expressed in angular size. The result of the integral, using the Bessel function of order one is given by:

$R\left(\theta_{\mathrm{s}}\right)=\frac{1}{2}-\frac{J_{1}\left(X\left(\theta_{\mathrm{s}}\right)\right)}{X\left(\theta_{\mathrm{s}}\right)}$,

where $X\left(\theta_{\mathrm{s}}\right)=\frac{2 \pi \theta_{\mathrm{s}} B}{\lambda}$. For the range of wavelength, baselines, and stellar radii considered here, this expression simplifies to: $R\left(\theta_{\mathrm{s}}\right)=\left(\frac{\pi \theta_{\mathrm{s}} B}{2 \lambda}\right)^{2}$.

The rejection factor is minimal when the planet is located on the first constructive fringe i.e. the separation between the planet and its parent is equal to $\theta_{\mathrm{s}-\mathrm{p}}=\frac{\lambda}{2 B}$. In the case of the Large Binocular Telescope (LBT) with its $14.4 \mathrm{~m}$ baseline, the first constructive fringe is at 11 milli-arcsec angular separation for $1.3 \mu \mathrm{m}$ wavelength, and consequently the optimum companion separation is $0.11 \mathrm{AU}$ for a system at $10 \mathrm{pc}$.

The efficiency of the null is also related to the stellar disk radius, $R_{\mathrm{S}}$, which depends on the stellar type as summarized in Table 1 using Bohm-Vitense \& Doyle (1989). The angular radius of an M 5 type star at $10 \mathrm{pc}$ is 0.12 milli-arcsec which is a factor 80 times smaller than the separation associated with the first constructive fringe observed at $1.3 \mu \mathrm{m}$. For such stellar radii and the range of separations considered here, the optimum rejection factor ranges from $10^{-2}$ to $10^{-4}$ and it would be below $5 \times 10^{-4}$ for separations greater than 35 times the star radius. The minimum separations to satisfy this condition for an M 5 star is 0.044 AU while the same efficiency is achieved for planets as far as $0.18 \mathrm{AU}$ separation from a Sun-like star, as summarized in Table 1.

In the particular cases considered here, the planet is not always on the first constructive fringe, and as a consequence there is some planet flux loss by transmission ranging from 0.1 to 1 as shown in Table 3. The improvement factor of the contrast ratio $\left(\frac{T_{\text {planet }}}{R}\right.$ i.e. ratio of the planet flux transmission to the rejection factor) ranges from $10^{2}$ to $8 \times 10^{3}$ for the selected planets in

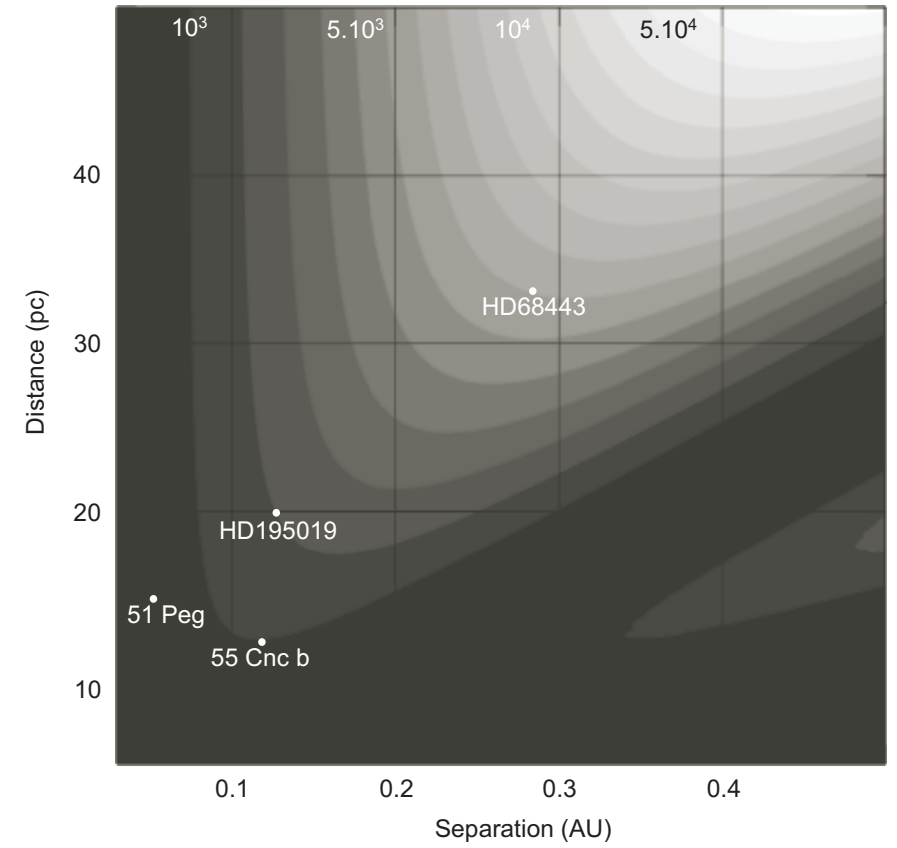

Fig. 2. The improvement factor of the contrast ratio $\left(\frac{T_{\text {planet }}}{R}\right.$ i.e. ratio of the planet flux transmission to the rejection factor) when using Bracewell Nulling at 1.3 micron with the LBT for a G0 type host star as a function of the planet-star separation and the distance of the system.

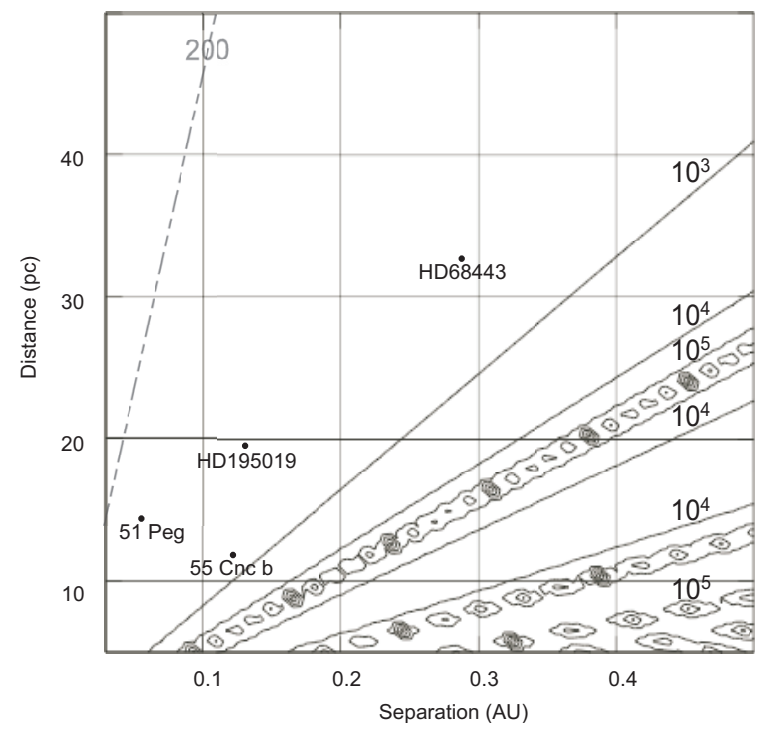

Fig. 3. The effective star-to-planet flux contrast when using Bracewell nulling with the LBT as a function of orbital separation and distance of the system. The phase value is equal to 0.3 , the planet radius is $1 R_{\mathrm{J}}$ and the primary star is of type G0.

Table 3 and is shown in Fig. 2 as a function of system distance and separation for G0 type stars.As a result the effective starto-planet ratio, including transmission loss, for 51 peg is 97 and for Tau boo is 127 as shown in Fig. 3 and in Table 2.

\section{Adaptive optics residual halo background}

Planet detectability is also affected by the halo of scattered light against which the planet must be detected, which comes 
Table 2. Detection parameters for selected known close-in extra-solar planets. The observing wavelength is $1.3 \mu \mathrm{m}, \mathrm{d}$ is the distance of the system, and $m_{v}$ and $R_{\mathrm{s}}$ are the host star $V$ magnitude and radius. $F_{V}$ and $F_{\mathrm{J}}$ are respectively the visible integrated photon flux from 0.65 to $0.95 \mu \mathrm{m}$ and the flux in $J$ band expressed in photons $\mathrm{m}^{-2} \mathrm{~s}^{-1} . R_{\mathrm{p}}$ is the planet radius inferred from Guillot et al. (1996), $\epsilon$ is the ratio of the planet flux to the star flux, and the last column represents the effective star-to-planet flux ratio when using Bracewell nulling with the LBT.

\begin{tabular}{|c|c|c|c|c|c|c|c|c|c|c|c|c|c|}
\hline Star & RA & Dec & $\begin{array}{c}d \\
(\mathrm{pc})\end{array}$ & $m_{v}$ & $\begin{array}{c}F_{V} \\
\times 10^{-8} \\
\end{array}$ & $\begin{array}{c}F_{J} \\
\times 10^{-8} \\
\end{array}$ & $\begin{array}{l}\text { Spec. } \\
\text { Typ. }\end{array}$ & $\begin{array}{c}R_{\mathrm{S}} \\
\text { (mas) }\end{array}$ & $\begin{array}{c}S \\
\text { (mas) }\end{array}$ & $\begin{array}{c}M_{\mathrm{p}} \sin i \\
\left(M_{\mathrm{J}}\right)\end{array}$ & $\begin{array}{c}R_{\mathrm{p}}^{a} \\
\left(R_{\mathrm{J}}\right)\end{array}$ & $\begin{array}{c}\epsilon \\
\times 10^{6} \\
\end{array}$ & $\frac{R}{\epsilon}$ \\
\hline 51Peg & 225728 & 204608 & 15.4 & 5.4 & 6.2 & 5.6 & G2 & 0.30 & 3.36 & 0.46 & 1.35 & 23 & 97 \\
\hline Ups And b & 013648 & 412420 & 16.5 & 4.6 & 13 & 12 & F7 & 0.34 & 3.55 & 0.68 & 1.4 & 19 & 132 \\
\hline $55 \mathrm{Cnc} b$ & 085236 & 281951 & 12.5 & 5.9 & 3.9 & 3.5 & G8 & 0.33 & 9.34 & 0.93 & 1.0 & 2 & 319 \\
\hline HD 130322 & 144733 & 001653 & 29.7 & 8.0 & 0.6 & 0.5 & K0 & 0.13 & 3.06 & 1.15 & 1.2 & 6 & 87 \\
\hline HD 217107 & 225816 & -022343 & 19.7 & 6.2 & 3.0 & 2.7 & G8 & 0.21 & 3.62 & 1.29 & 1.3 & 11 & 86 \\
\hline HD 195019 & 202819 & 184610 & 20.0 & 6.9 & 1.5 & 1.4 & G3 & 0.22 & 6.70 & 3.55 & 1.7 & 5 & 78 \\
\hline G186 & 021026 & -504925 & 12.0 & 6.1 & 3.2 & 2.9 & $\mathrm{~K} 1$ & 0.31 & 9.67 & 4.23 & 1.7 & 7 & 94 \\
\hline HD 68443 b & 081202 & -125307 & 33.0 & 6.9 & 1.5 & 1.4 & G5 & 0.13 & 8.71 & 7.18 & 1.7 & 1 & 108 \\
\hline HD 162020 & 175038 & -401906 & 31.3 & 9.1 & 0.2 & 0.2 & $\mathrm{~K} 2$ & 0.12 & 2.30 & 13.73 & 1.5 & 15 & 48 \\
\hline Tau Boo & 134716 & 172725 & 16.0 & 4.5 & 14 & 23 & F7 & 0.35 & 2.90 & 4.14 & 1.4 & 30 & 127 \\
\hline HD 38529 b & 054635 & 011005 & 42.4 & 5.9 & 3.9 & 3.5 & G4 & 0.11 & 3.01 & 0.77 & 1.0 & 2 & 173 \\
\hline HD 209458 & 220311 & 185304 & 47.0 & 4.3 & 17 & 15 & F8 & 0.11 & 1.0 & 0.63 & 1.35 & 29 & 106 \\
\hline HD 83443 b & 093712 & -431620 & 43.5 & 8.23 & 0.5 & 0.4 & K0 & 0.09 & 0.87 & 0.35 & 1.35 & 43 & 64 \\
\hline HD 46375 & 063101 & -665214 & 33.4 & 8.8 & 0.3 & 0.2 & K11 & 0.11 & 1.22 & 0.25 & 1.0 & 20 & 103 \\
\hline HD 187123 & 194658 & 342510 & 50.0 & 7.9 & 0.6 & 0.6 & G5 & 0.09 & 0.83 & 0.54 & 1.0 & 19 & 160 \\
\hline HD 68988 & 081822 & 612739 & 59.0 & 8.2 & 0.5 & 0.4 & G2 & 0.08 & 1.19 & 1.90 & 1.0 & 7 & 161 \\
\hline HD 108147 & 122546 & -640119 & 38.6 & 7.0 & 1.4 & 1.3 & F8 & 0.14 & 2.52 & 0.35 & 1.0 & 5 & 238 \\
\hline HD 168746 & 182150 & -115522 & 43.1 & 7.95 & 0.6 & 0.6 & G5 & 0.10 & 1.52 & 0.24 & 1.0 & 8 & 146 \\
\hline HD 75289 & 084740 & -414412 & 29.0 & 6.3 & 2.7 & 2.4 & G0 & 0.18 & 1.64 & 0.46 & 1.0 & 15 & 197 \\
\hline HD 179949 & 191533 & -241046 & 27.0 & 6.2 & 2.9 & 2.7 & F8 & 0.20 & 1.65 & 0.93 & 1.0 & 17 & 235 \\
\hline GJ 876 c & 225317 & -141549 & 4.7 & 10.2 & 0.1 & 0.1 & M4 & 0.34 & 27.4 & 0.56 & 1.0 & 2 & 420 \\
\hline HD 49674 & 065130 & 405204 & 40.7 & 8.1 & 0.5 & 0.5 & G0 & 0.12 & 1.46 & 0.12 & 1.0 & 9 & 196 \\
\hline HD 76700 & 085356 & -664804 & 59.7 & 8.9 & 0.3 & 0.2 & G5 & 0.07 & 0.83 & 0.19 & 1.0 & 15 & 144 \\
\hline
\end{tabular}

${ }^{a}$ Planet radius inferred from Guillot et al. (1996).

mainly from atmospheric turbulence. This contribution can be greatly reduced by using an adaptive optics system, but will result in a halo around the star with an intensity that depends on the amplitude of the residual errors and on the smallest spatial scale of the correction (Angel 1994). Detection of planets that are roughly $15 \mathrm{mag}$ fainter than their hosts, requires a halo $10^{4}$ times fainter than the central image peak, which can only be achieved by a high precision adaptive optics system with around 35 phase corrector actuators across each telescope aperture. As shown in Langlois et al. (2003), such a system is feasible and would be particularly efficient in a dual telescope configuration where very small spatial scale wavefront measurements can be combined with nulling capability. In such a configuration, the phase difference between the two telescopes is estimated using interferometric wavefront sensing techniques in which combining optics are arranged to deliver two equal intensity outputs at a wavefront sensing wavelength and nulling at the observing wavelength. This kind of recombination has already been achieved on a smaller scale by Hinz et al. (1999), where nulling occured around $10 \mu \mathrm{m}$ and the output at $2.2 \mu \mathrm{m}$ was used to tune the phase shift between the two apertures. For such adaptive optics system, we have analytically estimated the residual phase differences to be around $24 \mathrm{~nm}$ at $1.3 \mu \mathrm{m}$ for several known planet hosts in Table 3 used as guide stars. This could only be achieved by using multiple stage $\mathrm{AO}$ and/or modal control in order to minimize the low order residual errors responsible for image core widening. The previous calculation assumes a double $8.4 \mathrm{~m}$ aperture telescope, 0.7 arcsec seeing, wavefront sensing at 0.7 microns, and a $1 \mathrm{kHz}$ update rate by using Sandler et al. (1994) and Langlois (2001). This level of performance corresponds to a residual $1-2 \%$ transmission of the star flux when nulling and we predict that it will be unable to achieve the $10^{-3}$ level of nulling required here. Using the result from Angel (1994), we derive that the intensity of the residual halo is lower than the stellar intensity imperfectly subtracted by a factor ranging from 1 to 30 , as seen in Table 3 . These results were obtained by considering only closed-loop residual errors, not including scintillation and chromaticity effects which could be responsible, if uncompensated, for an additional 2-3\% Strehl loss (Fusco 2002).

\section{Detection limits with the LBT}

The detection method proposed here differs from previous approaches by associating partial dynamic Bracewell nulling with 
Table 3. Detectability of several known extra-solar planets with the LBT at $1.3 \mu \mathrm{m}$, where the fractional bandwidth is equal to 0.01 and the speckle lifetime is $\tau_{0}=1 \mathrm{~ms} . T$ is the exposure time for a $10-\sigma$ detection, $\frac{T_{\text {planet }}}{R\left(\theta_{\mathrm{s}}\right)}$ is the improvement factor of the contrast ratio, $T_{\text {Planet }}$ is the planet transmission, and $T_{\mathrm{ns}}$ is the integration time if the speckle fluctuations are negligible compared to both halo and leakage photon noise. We also give the exposure times at 1.2, 1.6, and $2.2 \mu \mathrm{m}$ and the full spectral bandwidth (Bwith) for detection in less than $5 \mathrm{~h}$. The quality of the wavefront assuming $N=35$ actuators per diameter is given by the residual wavefront error $=24 \mathrm{~nm}(\mathrm{rms})$ and the halo gain is $1.4 \times 10^{-4}$. If the speckle fluctuations were negligible, only $N_{\mathrm{m}}$ actuators would decrease the halo photo-electron counts to the leakage counts. COG is the center of gravity shift in arcseconds and $T_{\mathrm{COG}}$ is the exposure time for COG shift detection, assuming a bandwidth of 0.4 microns and that the astrometric accuracy is given by the ratio of the FWHM to the SNR.

\begin{tabular}{cccccccccccc}
\hline \hline Star & $\begin{array}{c}T_{\text {planet }} R\left(\theta_{\mathrm{s}}\right) \\
\times 10^{4}\end{array}$ & $T_{\text {Planet }}$ & $N_{\mathrm{m}}$ & $\begin{array}{c}T_{\mathrm{ns}} \\
\mathrm{min}\end{array}$ & $\begin{array}{c}T \\
(\mathrm{~h})\end{array}$ & $\begin{array}{c}T_{1.2 \mu \mathrm{m}} \\
G=1 \mathrm{E} 4\end{array}$ & $\begin{array}{c}T_{1.6 \mu \mathrm{m}} \\
G=2 \mathrm{E} 4\end{array}$ & $\begin{array}{c}T_{2.2 \mu \mathrm{m}} \\
G=4 \mathrm{E} 4\end{array}$ & $\begin{array}{c}\text { Bwith } \\
(5 \mathrm{~h})\end{array}$ & $\begin{array}{c}\text { COG } \\
\times 10^{3}\end{array}$ & $\begin{array}{c}T_{\text {COG }} \\
(\mathrm{h})\end{array}$ \\
\hline 51Peg & 22 & 0.3 & 9 & 0.02 & 0.01 & 0.01 & 0.01 & 0.01 & 5.3 & 10 & 9 \\
Ups And b & 25 & 0.3 & 8 & 0.12 & 0.2 & 0.2 & 0.2 & 0.2 & 3.9 & 8 & 8 \\
55Cnc b & 7.8 & 1.0 & 12 & 0.8 & 1.2 & 1.7 & 0.6 & 0.3 & 2.7 & 29 & 4 \\
HD 217107 & 9.7 & 0.3 & 11 & 0.5 & 0.5 & 0.6 & 0.5 & 0.5 & 3.0 & 14 & 12 \\
HD 195019 & 4.2 & 0.8 & 15 & 0.3 & 0.4 & 0.4 & 0.2 & 0.2 & 2.7 & 70 & 0.3 \\
HD 68443 b & 1.3 & 1.0 & 21 & 1.6 & 5.1 & 7.2 & 2.8 & 1.7 & 1.8 & 0.8 & 1.2 \\
Tau Boo & 38.0 & 0.2 & 7 & 0.1 & 0.1 & 0.1 & 0.1 & 0.1 & 4.0 & 5 & 25 \\
GJ 876 c & 8.5 & 1.0 & 12 & 63 & 2.9 & 4.7 & 27 & 148 & 0.2 & 65 & 70 \\
HD 130322 & 5.1 & 0.2 & 14 & 11 & 3.7 & 3.8 & 3.5 & 4.7 & 1.4 & 8.5 & \\
\hline
\end{tabular}

multi-spectral imaging or dynamic astrometric imaging. This approach allows to calibrate the still overwhelming stellar flux and to overcome the fact that the interferometer does not rotate. In this section we estimate the exposure time required to detect several known planet in such configurations.

Having estimated the various contributions to the background and the expected planet flux we now calculate the signal-to-noise ratio for broadband spectroscopy. If an integration yields a signal count of $C_{\mathrm{S}}$ photo-electrons per second from the planet and a background count $C_{\mathrm{B}}$, then the signal can be detected with the signal-to-noise ratio given by $S N R=\frac{C_{\mathrm{S}}}{\sqrt{C_{\mathrm{S}}+C_{\mathrm{B}}}}$. The background light level is dominated by the starlight leaking through the null and by the halo coming from the residual wavefront errors after the adaptive optics correction. The first contribution has a centrally peaked Airy pattern profile with a width of $1.22 \frac{\lambda}{D}$ shifted from the planet signal. The second contribution is spread over much greater radii and can be assumed to be constant. The optimum signal ratio may be obtained by weighting only the signal within the core of the planets central diffraction peak in a circle of diameter $\lambda / D$. The fraction of the signal flux in this solid angle is half the Strehl (St) and the signal from the planet in photoelectric counts is given by: $C_{\mathrm{S}}=F_{\mathrm{S}} \epsilon \frac{b}{h} S t\left(\frac{D}{2}\right)^{2} \pi \eta T$, where $F_{\mathrm{S}}$ is the star flux/ $\mathrm{Hz}, b$ is the fractional bandwidth, $D$ is the telescope diameter, $\eta$ is the product of the detector quantum efficiency and the optical transmission, and $T$ is the integration time. The background count, $C_{\mathrm{b}}$, can be written as the sum of the five terms corresponding to the leakage, diffuse residual halo, residual halo speckle, dark current, and read noise: $C_{\mathrm{B}}=C_{\text {leakage }}+C_{\text {halo }}+C_{\text {speckle }}+C_{\text {dark }}+C_{\text {read }}$, where $C_{\text {leakage }}=C_{\mathrm{S}} \frac{R\left(\theta_{\mathrm{s}}\right)}{\epsilon}, C_{\text {halo }}=C_{\mathrm{S}} \frac{\pi D}{4 G \epsilon}$, and $C_{\text {speckle }}=\left(\frac{C_{\mathrm{s}}}{G \epsilon}\right)^{2} \frac{\tau_{0}}{T}\left(\frac{2}{S t}\right)^{2}$ (Angel 1994), where $\tau_{0}$ is the speckle lifetime, or the exposure time required to obtain with adaptive optics correction independent realizations of residual speckles in the halo. This latter expression is similar to the speckle variance estimated in Racine et al. (1999). After adaptive optics compensation the speckle lifetime usually ranges from 1 to $100 \mathrm{~ms}$ (Ryan et al. 1998) and can in theory be reduced to $1 \mathrm{~ms}$ by incorporating temporal prediction in the wavefront reconstruction (Stahl \& Sandler 1995).

For several specific known objects from Marcy's almanac of extra-solar planets ${ }^{1}$ shown in Table 2, we estimate the exposure time for a 10- $\sigma$ detection. All the selected candidates with separations ranging from 0.05 to $0.3 \mathrm{AU}$ can be detected in less than ten hours at $1.3 \mu \mathrm{m}$ (Table 3 ). At this wavelength, the planet transmission is between $10 \%$ and $100 \%$, and both gain and rejection factors are high (within the $10^{-4}-10^{-3}$ range). In all cases, the detection is limited by the speckle fluctuation in the residual halo. The variance of the speckle is a few hundred times larger than the photon noise. If we consider that the speckle fluctuation can be reduced to a negligible amount compared to the halo and leakage photon noise, the integration time would be given by $T_{\mathrm{ns}}$ in Table 3 and all these extra-solar planets could be detected on minute timescales. In this case a number Nm of actuators ranging from 7 to 21 as shown in Table 3 would be sufficient to equalize halo and leakage photon noise. The integration time will in reality lie in between $T_{\mathrm{ns}}$ and $T$ according to the quality of the speckle smoothing and calibration and might be greater than $T$ if slow speckles due to non common path errors are present.

The residual speckle noise contribution can be significantly reduced by the use of multi-spectral or multi-polarization differential imaging. Marois et al. (2000) have shown that the technique can decrease the speckle noise by a factor $10^{4}$ by using three different wavelengths spanning the $\mathrm{CH}_{4}$ absorption band in the spectrum of brown dwarfs. This level is sufficient in all the cases considered here to reach the photon limit. Spectra

\footnotetext{
1 http://exoplanets.org/
} 


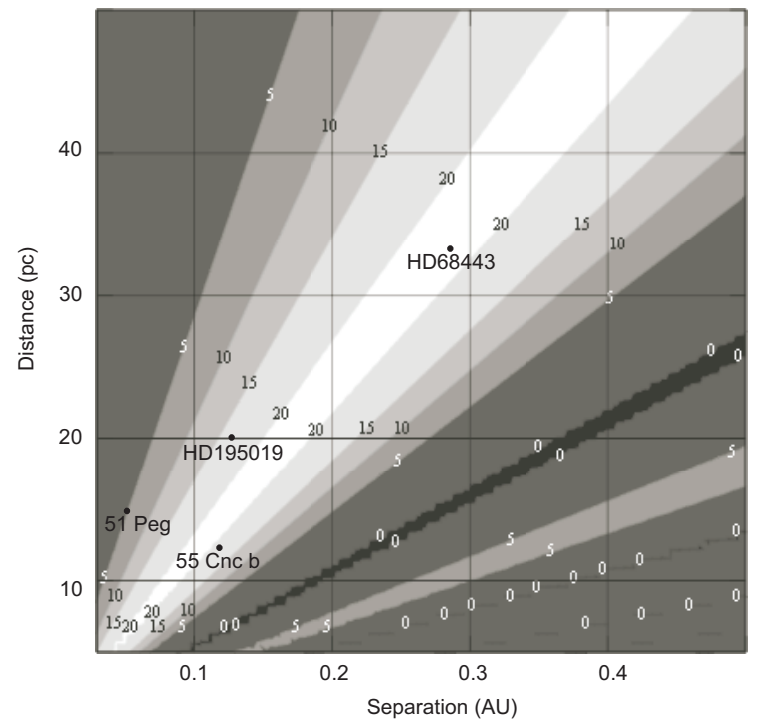

Fig. 4. COG shift in milli-arcseconds when using Bracewell nulling with the LBT at 1.3 micron for a G0 type host star as a function of the planet-star separation and the distance of the system.

for these planets are also predicted to have similar broad absorption bands between 1 and $3 \mu \mathrm{m}$ (the deepest band is centered around $1.3 \mu \mathrm{m}$ ) mainly due to water (Fig. 1). These water absorption lines could cause a decrease by a factor $10^{2}$ in the light reflected from the planet which might be used to reduce the speckle noise and also help distinguish the planet from residual starlight. With roughly a factor of 100 in flux between the planet and its host star, residuals after nulling and their slow variations need to be accurately calibrated out. This can be done by recording alternatively at two wavelengths with the null on and off. The accuracy required in this context could be reached when predicted deep absorption bands with contrasts greater than $10^{2}$ are present in the planet spectra. If this is not the case, another technique consists of estimating with a dynamic null the center of gravity displacement. When nulling is turned on, the center of gravity of the selected candidates (Table 2) moves from $10^{-4}$ to $5 \times 10^{-2}$ of the FWHM corresponding to 5-20 mas (Fig. 4). It has been shown in Currie et al. (2000) that $10 \mu$ as astrometric accuracy is achievable from the ground when using adaptive optics.

The accuracy of such astrometric methods is proportional to the ratio of the FWHM (33 milli-arcsec) to the signal-tonoise ratio. Assuming a bandwidth of 0.4 microns, the center of gravity motion could be detected in less than $50 \mathrm{~h}$ for 8 candidates, 2 of which (HD 195019 and Gl 86) could be detected in less than $1 \mathrm{~h}$. Both detection methods depend strongly on the phase value, itself dependent of the presence of clouds around the planet. A reasonable range would be $0.25-0.3$, leading to a range of exposure times up to a factor of 1.5 to 3 greater.

\section{Detection limits with the VLT and Keck}

If we consider a double aperture telescope configuration with longer baseline (e.g. the VLT $2 \times 8.2 \mathrm{~m}$ with $46.6 \mathrm{~m}$ baseline and Keck with a $85 \mathrm{~m}$ baseline) the efficiency of the null will be preserved as long as the quantity $\lambda R_{\mathrm{s}} / B S$ stays unchanged.

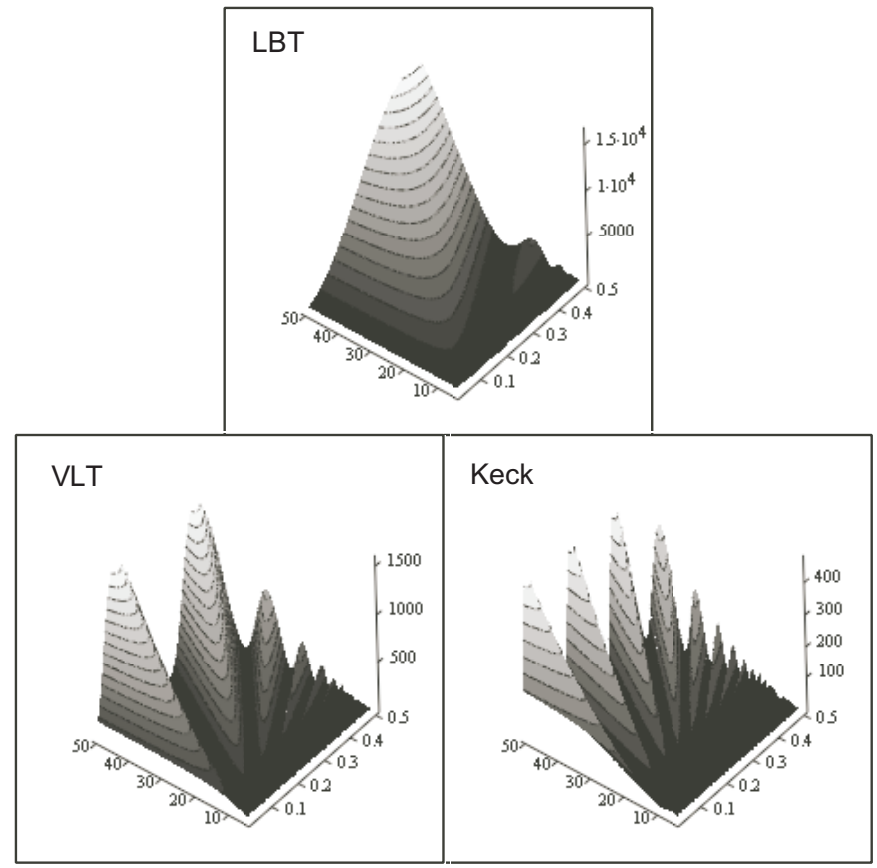

Fig. 5. The improvement factor of the contrast ratio when using Bracewell Nulling with LBT, VLT and Keck at 1.3 micron for a G0 type host star as a function of the planet-star separation and the distance of the system.

For the same wavelength domain the consequence is greater accessibility to closer targets as seen in Fig. 5. The null efficiency loss from the relative star diameter of a factor 10 for the VLT and a factor 100 for the Keck compared to the LBT, is compensated by an increase of the planet signal from the closer separations (Tables 3-5). The separation domains are 0.05-0.3 AU for the LBT and 0.03-0.1 AU for the VLT and the Keck. There is also a good complementarity between these telescopes; only 4 or 2 targets are observable by the LBT and the VLT or the Keck. When considering astrometry, the VLT is sensitive to separations ranging from 2 to 4 mas, with a total of 5 candidates detectable in less than $50 \mathrm{~h}$. For the Keck, the center of gravity shifts are only a few $\mu$ as and only one candidate (HD 209458) is detectable in $10 \mathrm{~h}$.

\section{Summary and conclusions}

Direct detection of reflected light from close-in extra-solar planets is achievable at near infrared wavelengths with short-baseline, large binocular interferometers equipped with adaptive optics and nulling. In this context nulling interferometry is able to achieve $10^{-3}$ or better suppression of the entire stellar Airy pattern with small loss of planet flux as close as $0.05 \mathrm{arcsec}$. The planet to star contrast ratio is predicted to be $10^{-4}-10^{-5}$ (Sudarsky et al. 2003) at $J$ and $H$ band, leading to integrations of a few minutes if photon noise of the residual star flux is the only limit. More realistically, due to "speckle" noise, integrations of several hours provide low-resolution spectra and access to possible absorption bands. Two detection methods may be used with similar efficiency: multi-wavelength imaging 
Table 4. Exposure time for detection of several known extra-solar planets with the VLT double aperture $8.2 \mathrm{~m}$ telescope and the shortest available baseline using both imaging and the center-of-gravity shift method. The range of the planet-star separations is $0.05-0.3 \mathrm{AU}$ and the assumptions and notation are the same as for Table 3. We also included the center-of-gravity shift (COG, in arcseconds) and the integration time for the COG method $\left(T_{\mathrm{COG}}\right)$.

\begin{tabular}{ccccc}
\hline \hline Star & $\begin{array}{c}T \\
(\mathrm{~h})\end{array}$ & $\begin{array}{c}B \text { with } \\
(\mu \mathrm{m})\end{array}$ & $\begin{array}{c}\text { COG } \\
\times 10^{3}\end{array}$ & $\begin{array}{c}T_{\mathrm{COG}} \\
(\mathrm{h})\end{array}$ \\
\hline 51Peg & 0.01 & 4.7 & 10 & 3 \\
HD 38529 b & 1.6 & 2.5 & 7 & 130 \\
HD 130322 & 0.2 & 2.3 & 13 & 50 \\
HD 217107 & 0.08 & 3.8 & 9 & 20 \\
HD 162020 & 0.05 & 2.5 & 26 & 5 \\
HD 209458 & 0.1 & 4.1 & 2 & 90 \\
Tau Boo & 0.01 & 4.9 & 10 & 1 \\
HD 83443 b & 0.1 & 2.5 & 3 & \\
HD 46375 & 0.2 & 2.8 & 4 & \\
HD 75289 & 0.1 & 3.3 & 4 & 120 \\
HD 179949 & 0.06 & 3.4 & 3 & 160 \\
HD 108147 & 0.4 & 2.6 & 5 & \\
HD 76700 & 1.5 & 2 & 1 & \\
\hline
\end{tabular}

Table 5. Exposure time for detection of several known extra-solar planets with the Keck double aperture telescope using both imaging and the center-of-gravity shift method. The range of planet-star separations is $0.03-0.1 \mathrm{AU}$ and the assumptions and notation are the same as for Table 3 .

\begin{tabular}{ccc}
\hline \hline Star & $\begin{array}{c}T \\
(\mathrm{~h})\end{array}$ & $\begin{array}{c}B \text { with } \\
(\mu \mathrm{m})\end{array}$ \\
\hline Ups And b & 2.2 & 0.5 \\
HD 217107 & 3.5 & 0.4 \\
HD 209458 & 0.06 & 4.6 \\
HD 168746 & 0.2 & 2.6 \\
HD 46375 & 0.05 & 2.8 \\
HD 187123 & 0.1 & 2.9 \\
HD 179949 & 0.05 & 3.7 \\
HD 68988 & 0.4 & 2.4 \\
\hline
\end{tabular}

and measurement of center-of-gravity shift. The first method is possible only when absorption bands are present, as predicted in most cases. Atmospheric correction with adaptive optics is also required for effective nulling and temporal averaging of "speckle" noise. This can be implemented on dual apertures interferometers. Given such systems, most of the close-in planets discovered by the radial velocity method with separations ranging from 0.05 to $0.3 \mathrm{AU}$ are detectable with the LBT. Longer baseline telescopes such as the VLT or Keck have the potential to detect slightly closer planets with separations between 0.03 and 0.1 AU. The sensitivity of these telescopes could in theory reach planets as small as Neptune.
Acknowledgements. The authors acknowledge J. R. P. Angel for fruitful conversations and aid during the course of this work.

\section{References}

Angel, J. R. P. 1994, Nature, 368, 203

Baudoz, P., Rabbia, Y., Gay, J., et al. 2000b, A\&AS, 145, 341

Beuzit, J.-L., Mouillet, D., Lagrange, A.-M., \& Paufique, J. 1997, A\&AS, 125, 175

Bohm-Vitense, E., \& Doyle, J. G. 1989, Introduction to Stellar Astrophysics - Vol.1 - Basic Stellar Observations and Data (Cambridge University Press)

Bracewell, R. N. 1978, Nature, 274, 780

Brown, R. A., \& Burrows, C. J. 1990, Icarus, 87, 484

Burrows, A., Guillot, T., Hubbard, W. B., et al. 2000, ApJ, 534, L97

Cameron, A., Horne, K., Penny, A., \& James, D. 1999, Nature, 402, 751

Currie, D., Tordo, S., Naesgarde, K., et al. 2000, ESO Photometric and Astrometric Analysis Program for Adaptive Optics, ASP Conf. Proc., 216, 381

Charbonneau, D., Noyes, R. W., Korzennik, S. G., et al. 1999, ApJ, 522, L145

Fusco, T. 2002, private communication

Guillot, T., Burrows, A., Hubbard, W. B., Lunine, J. I., \& Saumon, D. 1996, ApJ, 459, L35

Henry, G. W., Marcy, G. W., Butler, R. P., \& Vogt, S. S. 2000, ApJ, 529, L41

Hinz, P. M., Angel, J. R. P., Hoffmann, W. F., et al. 1998, Nature, 395, 251

Hinz, P. M., Angel, J. R. P., Woolf, N. J., Hoffmann, W. F., \& McCarthy, D. W. 1999, Imaging extra-solar systems from the ground: The MMT and LBT nulling interferometers, in ASP Conf. Ser., 194, 401

Hubbard, W. 1984, Planetary Interiors, Van Nostrand, New-York

Irvine, W. M. 1968, J. Atmos. Sci., 25, 610

Lane, A. P., \& Irvine, W. M. 1973, AJ, 78, 267

Langlois, M. P. 2001, Ph.D. Thesis, University of Paris 7, France

Langlois, M. P., Angel, J. R. P., Lloyd-Hart, M., Wildi, F., \& Love, G. 2003, PASP, submitted

Malbet, F. 1996, A\&AS, 115, 161

Marcy, G. W., \& Butler, R. P. 1997, Amer. Astron. Soc. Meet., 191, 7005

Marois, C., Doyon, R., Racine, R., \& Nadeau, D. 2000, PASP, 112, 91

Mayor, M., \& Queloz, D. 1995, Nature, 378, 355

Mayor, M., Queloz, D., Marcy, G., et al. 1995, IAU Circ., 6251, 1

Mazeh, T., Naef, D., Torres, G., et al. 2000, ApJ, 532, L55

Racine, R., Walker, G. A. H., Nadeau, D., Doyon, R., \& Marois, C. 1999, PASP, 111, 587

Roddier, F., \& Roddier, C. 1997, PASP, 109, 815

Rouan, D., Riaud, P., Boccaletti, A., Clénet, Y., \& Labeyrie, A. 2000, PASP, 112, 1479

Ryan, P. T., Fugate, R. Q., Angel, J. R. P., et al. 1998, Appl. Opt., 37, 7035

Sandler, D., Stahl, S., Angel, R., Lloyd-Hart, M., \& McCarthy, D. 1994, J. Opt. Soc. Am., 925, 945

Seager, S., Whitney, B. A., \& Sasselov, D. D. 2000, ApJ, 540, 504

Stahl, S. M., \& Sandler, D. G. 1995, ApJ, 454, L153

Sudarsky, D., Burrows, A., \& Pinto, P. 2000, ApJ, 538, 885

Sudarsky, D., Burrows, A., \& Hubeny, I. 2003, ApJ, 538, 885

Woolf, N. J., \& Angel, J. R. P. 1997, Planet finder options I: New linear nulling array configurations, in ASP Conf. Ser., 119, 285 